\title{
SEGUIMIENTO DE LOS SÓLIDOS EN SUSPENSIÓN DURANTE EL VACIADO DEL EMBALSE DE JOAQUÍN COSTA
}

\author{
C. Avendaño y R. Cobo. \\ Centro de Estudios y Experimentación de Obras Públicas (CEDEX).Paseo Bajo de la Virgen del Puerto, 3. \\ 28005-Madrid.
}

\section{RESUMEN}

Se han desarrollado dos métodos para medir Concentración de sólidos en suspensión "in situ" en pocos minutos. En concentraciones hasta $1,4 \mathrm{~g} / \mathrm{l}$, se utiliza un turbidímetro debidamente calibrado; para concentraciones superiores, se utiliza un matraz con el cuello calibrado para diferentes volúmenes de muestra. Estos métodos se ajustaron y calibraron en el Laboratorio de la División de Erosión y Sedimentología del Centro de Estudios Hidrográficos del CEDEX. Su empleo en el control de la concentración de sólidos en suspensión durante los vaciados del embalse de Joaquín Costa ha dado resultados satisfactorios en cuanto a operatividad y precisión.

Palabras clave: Vaciado de embalses, sedimentos en suspensión, medidas in situ, turbidímetro.

\begin{abstract}
Two methods have been developed to measure "insitu" suspended sediment in few minutes. A duly calibrated turbidimeter was used for concentrations less than $1.4 \mathrm{~g} / \mathrm{l}$. When higher concentration were involved, u flask was used, the neck of wich was calibrated for differentsampled volumes. These methods were udjusted and culibruted in the Erosion and Sedimentology Laboratory of the Hidragrafic Studies Center of CEDEX. Satisfactory results, u regards operability and accurracy, were obtained from its use in controlling suspenden solid concentrations, whenflushing the Joaquín Costa reservoir.
\end{abstract}

Key words: Reservoir flushing, suspended solids, in situ measurements, turbidity meter:

\section{INTRODUCCIÓN}

Los trabajos que el CEDEX ha desarrollado en las diferentes etapas del vaciado del embalse de Joaquín Costa (Barasona) han tenido diferentes objetivos:

- Realización de la batimetría del vaso del embalse para conocer la situación real del estado de aterramiento. Este estudio (CEDEX, 1994) permitió determinar que en diciembre de 1993 la capacidad del embalse era de $75.94 \mathrm{hm}^{3}$, el volumen de sólidos acumulados era de $24.764 \mathrm{hm}^{3}$ y que la cota de los mismos en la presa era de 418 m.s.n.m. Al finalizar el vaciado del embalse y entrar de nuevo en fase de explotación normal se realizó una nueva batimetría para obtener la nueva curva de capacidad. Los resultados permitieron evaluar que la capacidad del embalse en febrero de 1998 era de $84.798 \mathrm{hm}^{3}$ (CEDEX, $1998 \mathrm{~b}$ ).

- Realización del estudio sedimentológico de los materiales depositados en el embalse, que permitiera evaluar tanto su dragado como el posterior vaciado del mismo. Esta segunda fase necesitó de la puesta punto de una metodología adecuada las caraterísticas del sedimento (CEDEX, 1995 a). Esta etapa, realizada desde febrero a junio de 1994 se utilizó posteriormente para la siguiente etapa de evaluación (CEDEX, 1995; Avendaño et al. 1996 y 1997).

- Evaluación de la concentración de sólidos en suspensión a lo largo de un tramo de río posterior al embalse de Barasona durante los sucesivos vaciados que se resumen en la siguiente relación:

1. Oct. - Dic. 1995.- Evaluación de la concentración de sólidos en suspensión durante el 1 er vaciado (CEDEX, 1998 a).

2. Oct. - Nov. 1996.- Evaluación de la concentración de sólidos en suspensión durante el 2" vaciado (CEDEX, 1998 a).

3. Oct. 1997.-Evaluación de la concentración de sólidos en suspensión durante el $3 \underline{\text { er }}$ vaciado (CEDEX, I998 a). 


\section{CARACTERÍSTICAS DEL SEDIMENTO}

Los sedimentos depositados en el vaso del embalse tienen una textura media correspondiente a unos limos arcillosos, con un contenido medio de $65.87 \%$ de limo, un $26.56 \%$ de arcilla y un $5.57 \%$ de arena. En las proximidades de la presa el contenido en arcillas pasa a ser del $39.24 \%$, siendo el limo el $60.48 \%$ y quedando reducida la arena al $0.28 \%$. La densidad media del sedimento es de I $112 \mathrm{~kg} / \mathrm{m}^{3}$.

Desde el punto de vista de su composición el sedimento se puede definir como carbonatado con materiales detríticos. Considerando sólo los componentes mayoritarios (insolubles, carbonatos y materia volátil), la composición media del sedimento es de un $52.38 \%$ de insolubles (silicates s.1.), un $44.2 \%$ de carbonatos (caliza, dolomía, etc.) y un $3.42 \%$ de materia volátil. Presenta, además, pequeñas cantidades de otros elementos: $2.45 \%$ de $\mathrm{Fe}-1.16 \%$ de $\mathrm{Mg}$ $1.18 \%$ de $\mathrm{K}-0.15 \%$ de $\mathrm{Na}-0.04 \%$ de $\mathrm{P}$ y $0.03 \%$ de Mn. La composición mineralógica del sedimento esta constituida por un $15-25 \%$ de cuarzo, un $35-50 \%$ de calcita y un $30-50 \%$ de filisilicatos. Dentro de estos últimos se han determinado los siguientes minerales arcillosos: micas, caolinita, clorita, esmectita e interestratificados (esmectita y iclorita?).

\section{METODOLOGÍA}

Debido a la amplitud del rango de medida posible, y teniendo en cuenta la probabilidad de que se produjeran puntas de concentración muy por encima de lo previsto, se consideró necesario establecer dos sistemas de medida: uno para concentraciones bajas $(<1 \mathrm{~g} / 1)$ y otro para concentraciones superiores. En el primer caso se utiliza un turbidímetro debidamente ajustado y calibrado. Para el segundo, se puso a punto un sistema de "pesada en matraz". Se debe recalcar que esta metodología sólo es válida si el calibrado y ajuste se realizan con sedimentos del mismo tipo y naturaleza que los que se desean medir. Por tanto, los coeficientes de las ecuaciones empleadas en el cálculo de medidas de concentración utilizadas en este trabajo, sólo son válidas para el embalse de Joaquín Costa.

\section{Metodología para la evaluación de sólidos en suspensión con medidas nefelométricas.}

La turbidez del agua es producida por materias en suspensión tales como arcillas, compuestos orgánicos solubles, plancton y microorganismos. La turbidez es una expresión de la propiedad óptica que origina que la luz se disperse y absorba en vez de transmitirse en línea recta a través de la muestra.

La correlación de la turbidez con la concentración en peso de la materia en suspensión es difícil de establecer, ya que en la dispersión luminosa también intervienen el tamaño, la forma y el índice de refracción de las partículas.

Muchos turbidímetros comerciales, disponibles para medidas de turbidez baja, proporcionan datos comparativamente válidos sobre la intensidad de la luz dispersada en una dirección dada, predominantemente en ángulo recto a la luz incidente. Estos nefelómetros se ven escasamente afectados por las pequeñas variaciones de los parámetros de diseño y, por tanto, resultan especialmente Útiles como instrumentos estándar para medir turbideces bajas.

El método nefelométrico se basa en la comparación de la intensidad de luz dispersada por la muestra, en condiciones definidas, y la dispersada por una solución patrón de referencia en idénticas condiciones. Cuanto mayor es la intensidad de la luz dispersada, más intensa es la turbidez.

Un turbidímetro consiste en un nefelómetro con una fuente de luz para iluminar la muestra y uno o más detectores fotoeléctricos con un dispositivo de lectura exterior, para indicar la intensidad de la luz dispersada a $90^{\circ}$ de la vía de luz incidente (Fig. 1).

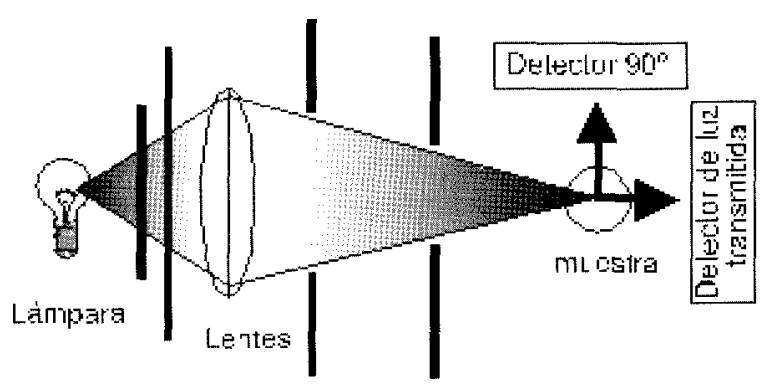

Figura1. Estructura interna del turbidímetro. Basics of turbidimeter. 
En el presente trabajo se ha utilizado un turbidímetro portátil $\mathrm{HACH}$, modelo $2100 \mathrm{P}$, con un rango de medida de 0.01 a $1000 \mathrm{NTU}$, divididas en tres rangos: de 0.01 a 0.99 ; de 10 a 99.99 y de 100 a 1000 NTU. El sistema Óptico incluye una lámpara con filamento de tungsteno, un detector de luz transmitida y un detector de luz dispersada a 90". El sistema compensa automáticamente las interferencias debidas al color y/o a la absorción de luz por los materiales y compensa las fluctuaciones de intensidad de la lámpara. El diseño del aparato se ajusta a los criterios especificados por la United States Environmental Protection Agencia, Method 180.1. Para realizar el calibrado del turbidímetro se utilizarón material extraído del propio embalse, de la zona próxima a la presa. Este material, una vez en el laboratorio, se dejó secar al aire y, después, fue sometido a un secado suave en estufa. A continuación se pesaron diferentes fracciones entre 0.1 y $1.3 \mathrm{~g}$ y se dejaron $48 \mathrm{~h}$ en 11 de agua para garantizar su saturación. De cada recipiente se obtuvieron 24 submuestras que se midieron con el turbidímetro. Con estos valores se realizo el gráfico de la figura 2, observándose una correlación lineal del tipo $\mathrm{y}=\mathrm{a}+\mathrm{bx}$, cuyas características son:

$\mathrm{N}^{\mathrm{o}}$ de observaciones: 168

Constante 'a' $=0.110356$

Coeficiente 'b' $=0.009263$

Error 's' de la estima $=0.054597$

Er. Std. Del coeficiente $=0.000098$

$r^{2}=0.9816 \quad r=0.991$

Ajustándose la recta de regresión de ecuación:

$$
\mathrm{Y}=0.110356+0.009263 \mathrm{X}
$$

Donde: $\quad \mathrm{Y}=$ Concentración de sólidos en suspensión en $\mathrm{g} / \mathrm{l}$ $\mathrm{X}=$ Lectura del turbidímetro en NTU

Como consecuencia de la experiencia del primer vaciado se decidió ampliar el rango de medida del turbidímetro, diluyendo la muestra en función de la concentración: $10 \mathrm{ml}$ de muestra en $100 \mathrm{ml}$ de agua destilada para valores inferiores a $1 \mathrm{~g} / \mathrm{l} \mathrm{y} 5 \mathrm{ml}$ de muestra en $250 \mathrm{ml}$ de agua destilada para concentraciones entre 1 y $10 \mathrm{~g} / 1$.

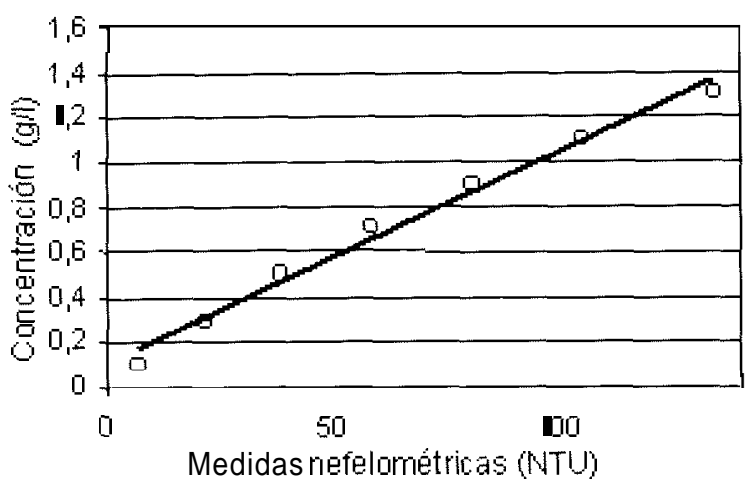

Figura 2. Rectas de calibración del turbidírnetro. Linear adjustments of the turbidimeter.

\section{Metodología para la evaluación de sólidos en suspensión con pesada de matraz}

Para concentraciones superiores a las indicadas en el apartado anterior, el turbidímetro no ofrece suficientes garantías de fiabilidad en la lectura, por lo que se hizo necesario desarrollar otro sistema de medida. Se procedió, en primer lugar, a encargar la fabricación de unos matraces especiales, con un cuello lo suficientemente largo como para que el contenido de una botella de muestreo quedara entre su parte inferior y superior. El cuello del matraz estaba graduado con precisión para poder leer directamente el volumen de la muestra, una vez vertida en el mismo. Se prepararon 168 muestras de valores comprendidos entre $2 \mathrm{~g} / \mathrm{l}$ y $80 \mathrm{~g} / \mathrm{l}$ de concentración, para volúmenes de 440,450,480 y $500 \mathrm{ml}$, que era el rango de medida de los matraces, obteniéndose una serie de rectas de regresión (Fig. 3), con las siguientes ecuaciones:

$$
\begin{array}{lll}
\mathrm{V}=440 \mathrm{ml} & \mathrm{P}=438.8806+0.275249 \mathrm{C} & \mathrm{r}=0.9998 \\
\mathrm{~V}=460 \mathrm{mi} & \mathrm{P}=458.8498+0.28607 \mathrm{C} & \mathrm{r}=0.9999 \\
\mathrm{~V}=480 \mathrm{ml} & \mathrm{P}=478.9701+0.299741 \mathrm{C} & \mathrm{r}=0.9997 \\
\mathrm{~V}=500 \mathrm{mi} & \mathrm{P}=498.2670+0.304179 \mathrm{C} & \mathrm{r}=0.9990
\end{array}
$$

donde:

$\mathrm{P}=$ peso de la muestra (agua + sedimento)

$\mathrm{C}=$ concentración de sólido

Debido a que en el momento del vaciado la obtención de resultados tenía que ser lo más rápi- 


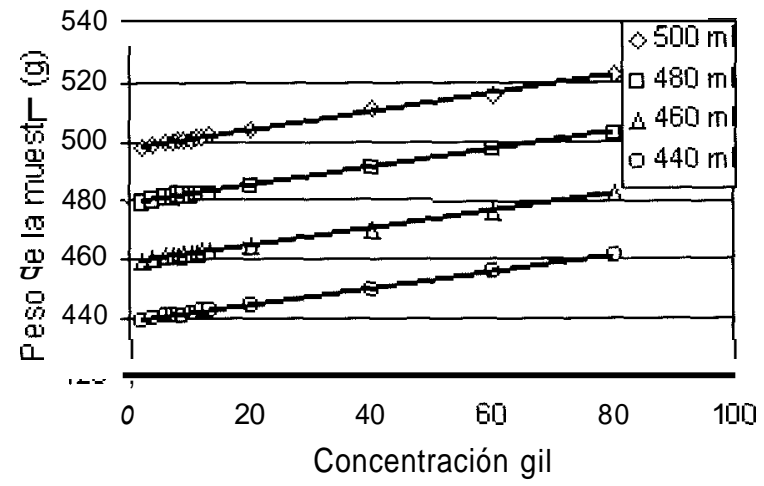

Figura 3. Rectas de calibrado del matraz. Regression lines for flask.

da posible, se desestimó la obtención de volúmenes de muestra constantes. Para poder calcular la concentración de sedimento en un volumen cualquiera de muestra, era necesario poder obtener la recta de regresión correspondiente a cualquier volumen. Para ello se obtuvieron las correspondientes rectas de regresión entre los parámetros 'a' y 'b' y el volumen de las muestras, obteniéndose las siguientes ecuaciones:

$$
\begin{array}{ll}
\mathrm{a},=2.78569+0.9913696 \mathrm{~V} & \mathrm{r}=0.9999 \\
\mathrm{~b}_{\mathrm{v}}=0.0552244+0.000502 \mathrm{~V} & \mathrm{r}=0.9831 \\
\text { donde: } &
\end{array}
$$

$\mathrm{V}=$ volumen de una muestra cualquiera

$\mathrm{a}=$ constante de la ecuación

$b_{v}=$ coeficiente de la ecuación

Se tiene, por tanto, para una muestra de volumen ' $\mathrm{V}$ ' cualquiera:

$$
\mathrm{P}=\mathrm{a}, \mathrm{b}, \mathrm{C} \text { de donde } \mathrm{C}=\left(\mathrm{P}-\mathrm{a}_{\mathrm{v}}\right) / \mathrm{b} \text {, }
$$

Para automatizar el proceso se desarrolló, en primer lugar un programa en GW-BASIC y, posteriormente, se implementó una hoja de cálculo EXCEL 97 (Fig. 4).

\section{PUNTOS DE CONTROL Y DE TOMA DE MUESTRAS}

Para poder seguir la progresión de los sólidos en suspensión, se establecieron tres puntos de control: el primero en el cauce del río Esera, en las inmediaciones de la presa (Pasarela); el segundo, en las proximidades de la confluencia

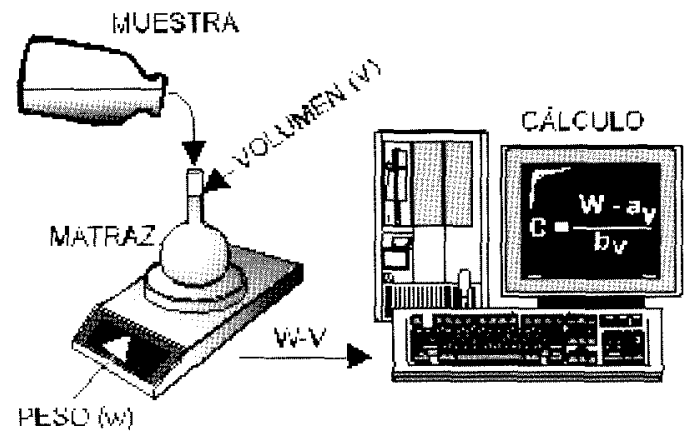

Figura 4. Proceso de medida con matraz. Flask calculation diagram.

del río Ésera con el río Cinca (Pte. de Olvena) y el tercero en el río Cinca, en el antiguo Puente de Las Pilas (Fig. 5).

Posteriormente, durante el vaciado, se realizaron también medidas en Monzón y Fraga. En estos puntos de control se tomaban dos muestras (botellas) mediante un turbisonda integradora en profundidad, suspendida mediante un cable desde un torno. De cada botella se obtienen cuatro submuestras en las que se mide la concentración de sólidos con el turbidímetro, dándose como valor la media de las ocho medidas efectuadas. En las medidas con el matraz, se analiza la totalidad de la muestra. Así mismo, cuando el valor de la concentración se encontraba en los límites de calibración (alta par el turbidímetro o baja para el matraz), las muestras se analizaban por ambos sistemas, dando como resultado la media. Durante el proceso de vaciado, sobre todo en el primero, se tomaron algunas muestras dobles con objeto de analizarlas, posteriormente, en el laboratorio y poder precisar la bondad del método. Las diferencias detectadas no superaron el $2.5 \%$.

En definitiva, para el segundo y tercer vaciado, se adoptó el siguiente criterio:

Medida de tanteo: dilución 10/100 ml en turbidímetro

En función del resultado obtenido, la hoja de cálculo indicaba el método de medida óptimo:

\begin{tabular}{ccc}
\hline Concentración & Dilución & Método \\
\hline $0-1 \mathrm{~g} / 1$ & 101100 & Turbidímetro \\
$\mathrm{I}-10 \mathrm{~g} / 1$ & 51250 & Turbidímetro \\
$10-15 \mathrm{~g} / 1$ & $5 / 250$ & Turbidímetro \\
$>15 \mathrm{~g} / 1$ & Muestra total & Matraz \\
\hline
\end{tabular}




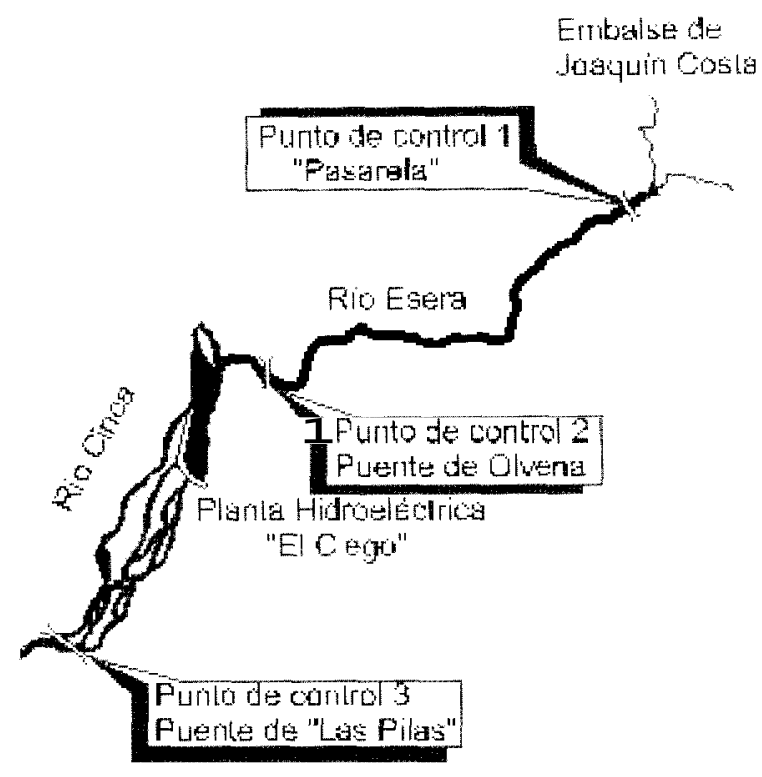

Figura 5. Situación de los puntos de control. Location of the selec ted check sections for sampling.

\section{DRAGADO 1994}

La necesidad de realizar un dragado del sedimento en la embocadura de los túneles de desagüe de fondo del embalse, se aprovecho para poner a punto la metodología y ajustar los parámetros de las ecuaciones de cálculo. Para ello, se tomaron muestras en el punto de control $\mathrm{n}^{\circ} 1$ (Pasarela), durante los meses de Febrero y Marzo. A partir de Abril se tomaron, además, muestras en la salida de las bombas y del túnel del aliviadero. Para que las muestras del bombeo fueran más representativas, se tomaba una botella de cada una de las bombas, tomando en total 9 botellas durante un periodo de 30 '. El valor de las concentraciones que se daba era la media de los valores obtenidos de las botellas correspondientes a cada bomba. Este procedimiento se siguió desde Febrero hasta Junio. Durante los meses de Febrero y Marzo la concentración media diaria se mantuvo por debajo de $1 \mathrm{~g} / \mathrm{l}$, excepto el $2 / 3(1.01 \mathrm{~g} / \mathrm{l})$, el $9 / 3(1 . \mathrm{I} \mathrm{g} / \mathrm{l})$ y el $23 / 3$ $(2.37 \mathrm{~g} / \mathrm{l})$. Durante los meses de Abril, Mayo y Junio la concentración media en la salida del túnel del aliviadero fue de $12 \mathrm{~g} / \mathrm{l}$, con una punto el $5 / 4$ de $44 \mathrm{~g} / 1$ y otras dos los días $11 / 5$ y $13 / 5$ de
$26.45 \mathrm{~g} / \mathrm{l}$ y $32.97 \mathrm{~g} / \mathrm{l}$, respectivamente. A la vista de estos valores se realizaron unas tomas en el punto de control $\mathrm{n}^{\circ} 2$, Pte. de Olvena, obteniéndose siempre valores muy inferiores a $1 \mathrm{~g} / \mathrm{l}$.

\section{PRIMER VACIADO (1995)}

El primer vaciado dio comienzo el día 12 de Octubre a las $3 \mathrm{~h}$ y duró hasta las $20 \mathrm{~h}$ de ese mismo día. Como se puede observar en la figura 6 , mientras duró la salida de agua, la concentración de sólidos en suspensión en el punto de control $\mathrm{n}^{\circ} 1$, se mantuvo por debajo de $1 \mathrm{~g} / \mathrm{l}$. La última muestra que se pudo tomar, instantes antes de que la colada de barro anegara el cauce del río Esera, fue de $492 \mathrm{~g} / \mathrm{l}$. En este punto no se pudo volver a hacer un muestreo hasta el día 18 de Octubre. En todo momento la concentración fue muy elevada ( $>40 \mathrm{~g} / \mathrm{l})$.

En el 2" punto de control, Pte. de Olvena, la concentración se mantuvo por debajo de $1 \mathrm{~g} / \mathrm{l}$ hasta las $22 \mathrm{~h}$ del día 12, dos horas después de que en Pasarela el cauce quedara totalmente anegado. En ese momento, la masa de agua que anegaba el cauce avanzaba a pocos centímetros por minuto de velocidad y, sobre ella, circulaba el agua del río Esera, que atravesaba el embalse y salía por el túnel de la margen izquierda. Desde las 22 h del día I2 y hasta las $2 \mathrm{~h}$ del día 13, el agua se fue encajando, tanto en los sedimentos del vaso, como en los depositados en el cauce; paralelamente, la concentración de sólidos fue aumentando hasta alcanzar los $76 \mathrm{~g} / \mathrm{l}$. Aproxima-

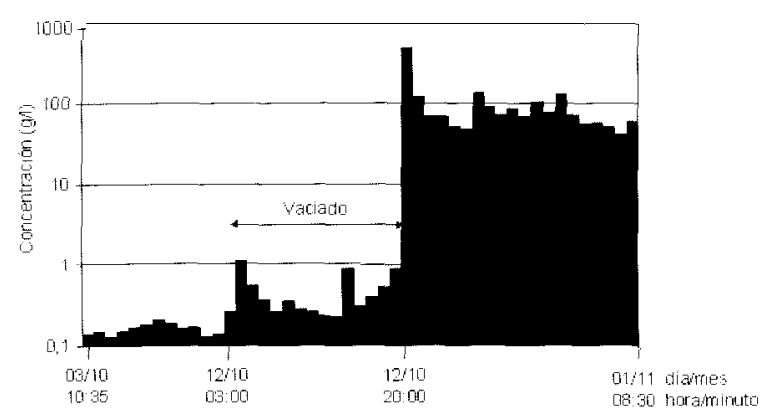

Figura 6. Vaciado de 1995. Concentración de sólidos en suspensión en el punto de control $\mathrm{n}^{\circ} \mathrm{l}$ (Pasarela, río Esera). Dam flushing of 1995. Concentration of suspended solids in the check point $n^{\circ} 1$ (Pasarela, river Ésera). 
damente a la 19 h del día 13 , la masa de lodo llegó al punto de control $\mathrm{n}^{\circ} 2$ anegándolo. La última muestra tomada en este punto tenía $282 \mathrm{~g} / \mathrm{l}$.

En el punto de control $\mathrm{n}^{\circ} 3$, Pte. de Las Pilas, en el río Cinca, la concentración se mantuvo por debajo de $1 \mathrm{~g} / \mathrm{l}$ hasta el día 13 a la $17 \mathrm{~h}$. A partir de ese momento la concentración aumentó rápidamente, de forma que a las 19:30 h era de $8 \mathrm{~g} / \mathrm{l}$ y a la $22 \mathrm{~h}$ de $41 \mathrm{~g} / \mathrm{l}$. A las $22: 45 \mathrm{~h}$ se midieron $85 \mathrm{~g} / \mathrm{l}$, produciéndose, a continuación, un paulatino descenso hasta que, bruscamente, se registró un nuevo aumento (Fig. 7). Este esquema se repitió varias veces, aunque de manera irregular. El proceso antes descrito, puede atribuirse al efecto de encajamiento del río en el sedimento del embalse y al desplome de grandes bloques de sedimento, al inestabilizarse los taludes creados por el agua. El gran aumento de concentración que se produce a partir del día 12/11 se debe a un gran aumento en el caudal del Ésera, que pasó de $22 \mathrm{~m}^{3} / \mathrm{s}$ a $69 \mathrm{~m}^{3} / \mathrm{s}$, con puntas de $90 \mathrm{~m}^{3} / \mathrm{s}$, debido a las lluvias que se produjeron días antes. Así mismo, se produjeron precipitaciones el día 18/11 y fuertes tormentas el 22/11. A partir del día 28/11, la concentración empezó a disminuir debido a que el río ya había creado un cauce, más o menos estable, en los sedimentos del vaso. El día $14 / 12$ se cerraron las compuertas del embalse y el día 15/12 se dejó de muestrear.

\section{SEGUNDO VACIADO (1996)}

El segundo vaciado dio comienzo el día 1 de Octubre a las 12:15 h. Anteriormente, los días

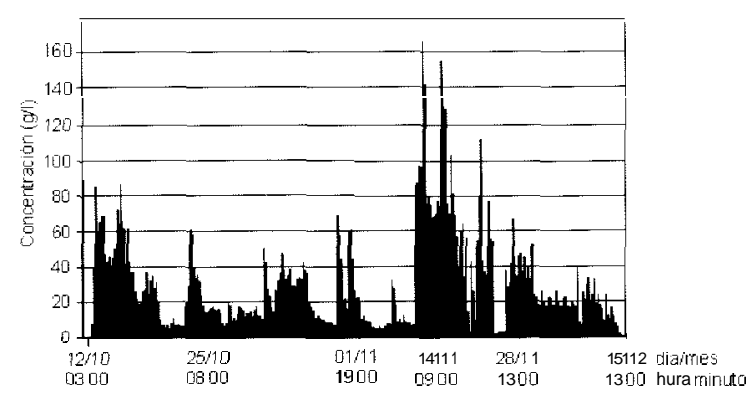

Figura 7. Vaciado de 1995.Concentración de sólidos en suspensión en el punto de control $n^{\circ} 3$ (Puente de las Pilas, río Cinca). Dam flus'hing of 1995. Concentration of suspended solids in the check point $n^{\circ} 3$ (Las Pilas Bridge, river Cinca).

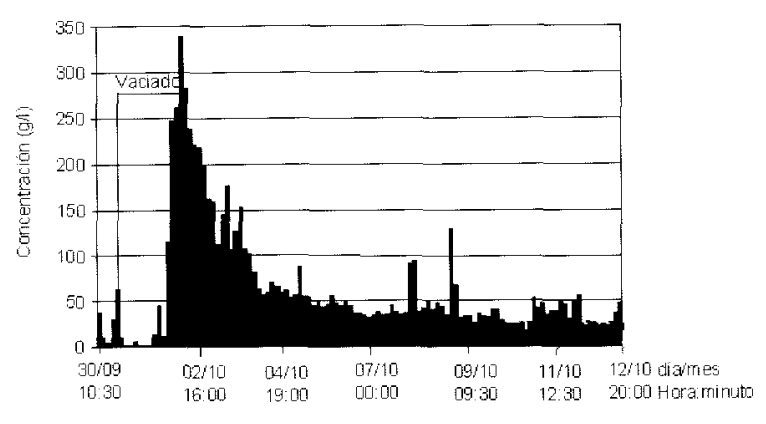

Figura 8.- Vaciado de 1996. Concentración de sólidos en suspensión en el punto de control $n^{\circ} 1$ (Pasarela, río Ésera). Dam flushing of 1996. Concentration of suspended solids in the check point $n^{\prime \prime} I$ (Pasarela, niver Ésera).

30/9 y 1/10, se habían tomado muestras de las pérdidas que tenían las compuertas del túnel de la margen izquierda, en el punto de control $\mathrm{n}^{\mathrm{o}} 1$, Pasarela, obteniéndose valores de $37.63 \mathrm{~g} / \mathrm{l}$ y $63.25 \mathrm{~g} / \mathrm{l}$, respectivamente. Cuando comenzó a salir el agua, la concentración bajó drásticamente hasta llegar a valores de $0.4 \mathrm{~g} / \mathrm{l}$; manteniéndose a niveles bajos durante todo el día 1 y parte del siguiente (Fig. 8). El día 2 a las $8 \mathrm{~h}$, la concentración empezó a aumentar conforme disminuía el agua del embalse. Así, en una hora, la concentración aumentó un orden de magnitud, pasando de II $\mathrm{g} / \mathrm{l}$ a las $10 \mathrm{~h}$, hasta $\mathrm{I} 14 \mathrm{~g} / \mathrm{l}$ a la $11 \mathrm{~h}$. A las I 1:45 h la concentración era de $247 \mathrm{~g} / \mathrm{l}$; a las $12 \mathrm{~h}$, de $262 \mathrm{~g} / \mathrm{l}$. En este momento terminó el vaciado pero, al contrario que el año anterior, no se interrumpió el curso de agua, debido a que todavía se mantenía el cauce excavado por el río en los sedimentos del embalse. Inmediatamente después del vaciado se alcanzó el mayor nivel de concentración: $339 \mathrm{~g} / \mathrm{l}$. A partir de este momento, la concentración de sólidos disminuyó rápidamente, de manera que el día 4 a las 9:30 h llega a $80 \mathrm{~g} / \mathrm{l}$. Desde este día y hasta el día 6, el descenso en los valores de concentración es más suave, estabilizándose en torno a los $30-35 \mathrm{~g} / \mathrm{l}$; valor que se mantuvo, con irregularidades, hasta que se dejó de muestrear es esta sección, el día 12/10 (Fig. 8).

En el Pte. de Olvena, al no haberse producido, como en el primer vaciado, un aterramiento del cauce, los acontecimientos estuvieron casi sincronizados con los sucesos de Pasarela. En un principio se midieron concentraciones con valores bajos, que fueron aumentando progresivamente, hasta que el día 2 a las 13:45 h, se midie- 


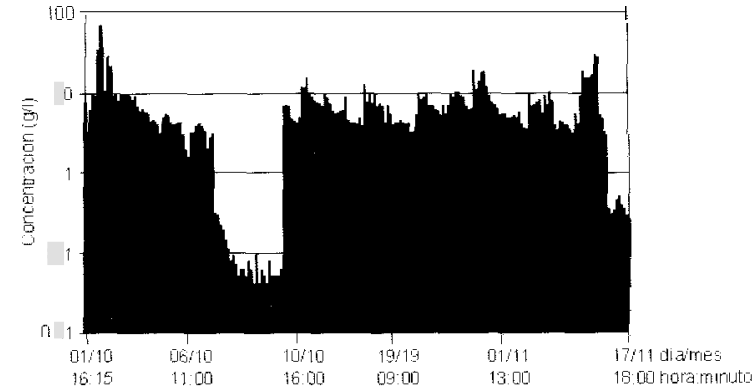

Figura 9.- Vaciado de 1996. Concentración de sólidos en suspensión en el punto de control $n^{\circ} 3$ (Puente de las Pilas, río Cinca). Dam flushing of 1996. Concentration of saspended solids in the check point $n^{\circ} 3$ (Las Pilas Bridge, river Cinca).

ron $254 \mathrm{~g} / 1$. En este momento la sección comenzó a aterrarse, generándose, incluso, corrientes que progresaban aguas arriba debido a la represa que ocasionaba la reja para recogida de peces. En estas circunstancias la representatividad del muestreo no se podía garantizar y se suspendió el mismo. En el puente de Las Pilas la concentración no superó los $10 \mathrm{~g} / \mathrm{l}$ hasta el día $11 / 10$ a las 8:00 h $(12.02 \mathrm{~g} / 1)$. A partir de este instante se repite el esquema antes descrito de subidas bruscas de concentración, seguidas de descensos más o menos suaves (Fig.9).

\section{TERCER VACIADO (1997)}

El tercer desembalse comenzó el día 3 de Octubre a las $12 \mathrm{~h}$ y terminó el día siguiente a las

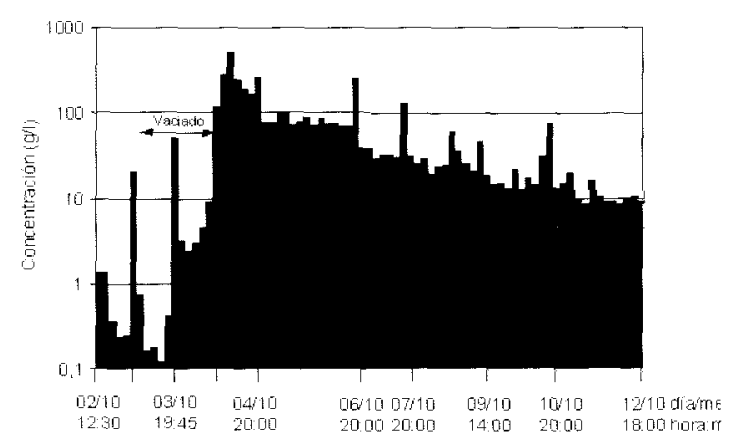

Figura 10.- Vaciado de 1997. Concentración de sólidos en suspensión en el punto de control $n^{\circ}$ I(Pasarela, río Ésera). Dam flushing of 1997. Concentration of suspended solids in the check point $n^{\circ} 1$ (Pasarela, river Ésera).
$13 \mathrm{~h}$. En el punto de control $\mathrm{n}^{\circ} 1$, durante las primeras horas, se midieron concentraciones por debajo de $1 \mathrm{~g} / \mathrm{l}$. Cuando salió toda el agua y se estableció un régimen fluvial, la concentración se elevó rápidamente. Entre las $13 \mathrm{~h}$ y las $14 \mathrm{~h}$, la concentración pasó de $9 \mathrm{~g} / \mathrm{l}$ a $500 \mathrm{~g} / \mathrm{l}$; a continuación, descendió progresivamente hasta estabilizarse en torno a $70-80 \mathrm{~g} / \mathrm{l}$. A partir del día 6 de Octubre y hasta el final del muestreo, el día 10, la concentración descendió de forma lenta, pero continua, aunque con valores puntuales de cierta importancia, que también tendían a ser menores con el tiempo (Fig. 10).

En el punto de control $n^{\circ} 2$, debido al escaso valor de los datos obtenidos, sobre todo en el segundo vaciado, se decidió no muestrear en este tercer año, concentrhndose los trabajos en los puntos de control 1 y 3 .

Durante el tiempo transcurrido entre el $2 "$ y el $3^{\text {er }}$ vaciado, el antiguo puente de "Las Pilas" había sido derruido y sustituido por uno nuevo, desde el que no era posible realizar la toma de muestras; por este motivo el muestreo se realizó, en su totalidad, a la salida del azud de la Central de Hidro-Nitro.

El efecto del desembalse se reflejó dos horas $\mathrm{y}$ inedia después que en el punto $\mathrm{n}^{\mathrm{o}} \mathrm{I}$. A las 15:30 h se midieron $23.62 \mathrm{~g} / 1$, que fue el máximo medido. Rápidamente la concentración disminuyó, estabilizhndose en torno a valores de 2 $3 \mathrm{~g} / \mathrm{l}$, aunque con bruscos aumentos que se fueron suavizando y espaciando con el tiempo (Fig.1I).

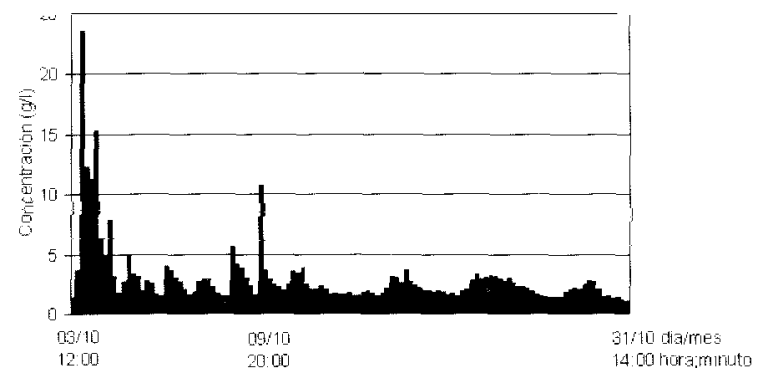

Figura 11.- Vaciado de 1997. Concentración de sólidos en suspensión en el punto de control $\mathrm{n}^{\mathrm{o}} 3$ (Toma del canal de la Central Arias 1). Dam flushing of 1997. Concentration of suspended solids in the check point n'3 (Hydropower Arias I chamel). 


\section{BIBLIOGRAFÍA}

AVENDAÑO, C., R. COBO, E. SANZ Y J.L. GÓMEZ. 1996. Metodología para la evaluación de sólidos en suspensión “in situ”. Aplicación en el dragado del embalse de Barasona (Huesca). Revista de Ingeniería Civil, 102: 123-128.

AVENDAÑO, C., R. COBO, E. SANZ \& J.L. GÓMEZ. 1997. Evaluation of suspended solids "in situ". Application to the dredgin ans flushing of the Barasona Reservoir. Nineteenth International Congress on Large Darns. Florencia, vol. III: 825-834.

CEDEX, 1994. Reconocimientos batimétricos de embalses. Embalse de Barasona. Informe para la Dirección General de Obras Hidráulicas (MOPTMA). 27 pp, 4 anexos, 19 planos.

CEDEX, 1995 a. Reconocimientos sedimentológicos de embalses. Embalse de Barasona. Informe para la Dirección General de Obras Hidráulicas (MOPTMA), 144 pp.
CEDEX, 1995 b. Vaciado del embalse de Barasona (1994-1996). Control de la concentración de sólidos en suspensión durante el dragado del embalse. Informe para la Dirección General de Obras Hidráulicas (MOPTMA), 114 pp.

CEDEX, 1998 a. Vaciado del embalse de Barasona (1995-1997). Evaluación de la concentración de sólidos en suspensión de los vaciados del embalse (199.5, 1996 y 1997). Informe para el Ministerio de Medio Ambiente. Secretaría de Estado de Aguas y Costas. Dirección General de Medio Ambiente y Calidad de las Aguas, 90 pp

CEDEX, 1998 b. Vaciado del embalse de Barasona (I99.5-1997). Reconocimiento batimétrico (1998). Informe para el Ministerio de Medio Ambiente. Secretaría de Estado de Aguas y Costas. Dirección General de Medio Ambiente y Calidad de las Aguas, 40 pp, anejos, 19 planos. 